\title{
Gerador de alta frequência no tratamento de fasciíte necrotizante em pessoa com diabetes
}

\section{High-frequency generator in the treatment of necrotizing fasciitis in a person with diabetes}

\section{Generador de alta frecuencia en el tratamiento de la fascitis necrotizante en persona con diabetes}

\author{
Uiara Aline de Oliveira Kaizer ${ }^{1,2^{*}}$, Marcelle Hauany Silva Costa ${ }^{3}$, Elaine Aparecida Rocha \\ Domingues 4 , Maiume Roana Ferreira de Carvalho ${ }^{3}$
}

ORCID IDS

Kaizer UAO (D) https://orcid.org/0000-0002-9115-8043 Costa MHS (D) https://orcid.org/0000-0003-1280-691X Domingues EAR (D) https://orcid.org/0000-0002-7589-2344 Carvalho MRF (DD https://orcid.org/0000-0002-0324-7506

\section{COMO CITAR}

Kaizer UAO; Costa MHS; Domingues EAR; Carvalho MRF (2019) Gerador de alta frequência no tratamento de fasciíte necrotizante em pessoa com diabetes. ESTIMA, Braz. J. Enterostomal Ther., 17: e1719. https://doi.org/10.30886/estima.v17.688_PT

\section{RESUMO}

Objetivo: Relato de caso sobre o tratamento da fasciíte necrotizante com a utilização do gerador de alta frequência (AF) em uma pessoa com diabetes. Métodos: Trata-se de um estudo descritivo de natureza aplicada, desenvolvido em um ambulatório no interior de Minas Gerais. A paciente foi submetida a sessões de curativo durante três vezes na semana para aplicação de alta frequência. A cicatrização foi avaliada semanalmente por meio do instrumento Pressure Ulcer Scale Healing (PUSH) e pelo software Image J. Resultados: Houve redução significativa da área da lesão, que foi mensurada durante seis avaliações semanais. Nas quatro primeiras avaliações, foi obtida redução progressiva da lesão em 11,78 cm², ou seja, $90 \%$ da área. Conclusão: O uso do gerador de AF foi efetivo no tratamento da fascí́te necrotizante, com redução da área da lesão.

DESCRITORES: Diabetes mellitus. Cuidados de Enfermagem. Avaliação em Enfermagem. Cicatrização. Estomaterapia.

1.Prefeitura de Sorocaba - Sorocaba/SP - Brasil

2.Universidade Estadual de Campinas - Programa de Pós-Graduação da Enfermagem - Campinas/SP - Brasil

3.Universidade Vale do Rio Verde - Curso de Enfermagem - Três Corações/MG - Brasil

4.Universidade Estadual de Campinas - Campinas/SP - Brasil

Autora correspondente: viara_oliveira@hotmail.com

Recebido: Jan. 22, 2019 | Aceito: Ago. 28, 2019 


\section{ABSTRACT}

Objective: A case report on the treatment of necrotizing fasciitis with the use of high frequency (HF) generator in a person with diabetes. Methods: This is descriptive study of applied nature developed in an outpatient clinic in Minas Gerais. The patient underwent dressing sessions three times a week for high frequency application. Healing was weekly evaluated using the Pressure Ulcer Scale Healing (PUSH) instrument and Image J software. Results: The area was significantly reduced, which was measured during six weekly evaluations. In the first four evaluations, a progressive reduction of the lesion was obtained in $11.78 \mathrm{~cm}^{2}$, that is, $90 \%$ of the area. Conclusion: The use of the AF generator was effective in the treatment of necrotizing fasciitis, reducing the lesion area.

DESCRIPTORS: Diabetes mellitus. Nursing care. Nursing evaluation. Healing. Stomatherapy.

\section{RESUMEN}

Objetivo: Relato de caso sobre el tratamiento de la fasciitis necrotizante con la utilización del generador de alta frecuencia (AF) en una persona com diabetes. Métodos: Se trata de un estudio descriptivo de naturaleza aplicada, desarrollado en un ambulatorio en el interior de Minas Gerais. La paciente fue sometida a sesiones de curativo durante tres veces a la semana para aplicación de alta frecuencia. La cicatrización fue evaluada semanalmente a través del instrumento Pressure Ulcer Scale Healing (PUSH) y área de la lesión por el software Image J. Resultados: Hubo una reducción significativa en el área de la lesión, que se midió durante seis evaluaciones semanales. Durante las cuatro primeras evaluaciones se obtuvo una reducción progresiva de la lesión en 11,78cm², es decir, el 90\% del área. Conclusión: El uso del generador de AF fue efectivo en el tratamiento de la fascitis necrotizante, con reducción del área de la lesión.

DESCRIPTORES: Diabetes mellitus. Cuidados de enfermería. Evaluación en enfermería. Cicatrización. Estomatoterapia.

\section{INTRODUÇÃO}

O diabetes mellitus é uma das principais doenças crônicas que acometem a população mundial e vem se tornando um problema de saúde pública. No Brasil, estimativas constataram que 11,9 milhões de indivíduos, na faixa etária entre 20 e 79 anos possuem a doença, classificando o país como o quarto no ranking de diabetes mellitus no mundo ${ }^{1}$. Diversas são as complicações decorrentes da doença, entre elas as macrovasculares, microvasculares, neuropatias por maior suscetibilidade ao risco de infecções ${ }^{2}$.

A fasciíte necrotizante é uma infecção rara e grave caracterizada por necrose extensa e rapidamente progressiva. Foi descrita em 1871 pelo cirurgião Dr. Joseph Jones, todavia, o termo fasciíte necrotizante passou a ser utilizado por Wilson Ben a partir de 1952, para descrever sua principal característica: a necrose do tecido celular subcutâneo mantendo preservado o músculo subjacente. Sua incidência é maior em indivíduos adultos com fatores de risco predisponentes, tais como diabetes tipo 2, idade avançada, câncer, doença vascular periférica e acometidos por traumas abertos e fechados. É caracterizada por necrose extensa que pode acometer a fáscia superficial de tecidos moles, a parede abdominal, membros e períneo, por meio de trombose venosa de pequenos vasos e supuração. Seu tratamento deve ser precoce, por meio do uso de antibióticos de amplo espectro, desbridamento cirúrgico agressivo e medidas de suporte clínico e nutricional ${ }^{2}$.

A prática de cuidados aos pacientes com feridas é uma especialidade dentro da enfermagem, reconhecida pela Sociedade Brasileira de Enfermagem em Dermatologia (Sobende) e pela Associação Brasileira de Estomaterapia (Sobest) ${ }^{3,4}$. Essa prática é uma responsabilidade atribuída ao enfermeiro que deve avaliar a lesão e prescrever o tratamento adequado. Atrelado a esse fato, a Lei Federal no 7.498 de 25 de junho de $1986^{5}$, o Decreto Federal no 94.406 de 08 de junho de $1987^{6}$ e a Resolução do Conselho Federal de Enfermagem (Cofen) no 358 de 15 de outubro de $2009^{7}$ dispõem sobre a Sistematização da Assistência de Enfermagem (SAE); a Resolução Cofen no 567/2018 regulamenta a atuação da equipe de enfermagem no cuidado aos pacientes com feridas, dispondo autonomia para participação na avaliação, elaboração de protocolos, seleção e indicação de novas tecnologias na prevenção e tratamento de lesões ${ }^{8}$.

Dessa forma, o profissional de enfermagem é o responsável pelo planejamento dos cuidados à pessoa com ferida, por meio de métodos terapêuticos e junto à equipe interdisciplinar; tem o objetivo de ajudar na cicatrização da ferida sem complicações, com a restauração das funções em menor tempo possível e a prevenção das sequelas ${ }^{9}$.

Entre a utilização de novas tecnologias no tratamento de lesões destaca-se a utilização da estimulação elétrica. 
Estudos indicam que a estimulação elétrica é uma boa opção para auxiliar na melhoria no processo de cicatrização por promover a migração de células epiteliais, macrófagos e neutrófilos para a lesão. Além disso, a estimulação elétrica altera o DNA e resulta em maior síntese de colágeno pelos fibroblastos e, dessa forma, potencializa a cicatrização ${ }^{10}$.

O gerador de alta frequência (AF) é um aparelho que opera com correntes elétricas alternadas de alta e baixa intensidades, com tensão aproximada de 30 a 40 mil volts e frequência de 150 a $200 \mathrm{kHz}$. Possui eletrodos de vidro que contêm ar rarefeito (vácuo) ou gás (neônio, xenônio ou argônio) em seu interior. A passagem da corrente elétrica através do aparelho provoca ionização das moléculas de gás que se tornam fluorescentes devido ao grande impacto energético. Ao entrar em contato com o eletrodo, a pele promove um faiscamento que transforma o oxigênio em ozônio ${ }^{11}$.

O ozônio foi descoberto no ano de 1834, por Christian Friedrich Schönbein, que o descreveu como uma substância oxidante e desinfetante. Em 1857, o químico Werner Von Siemens desenvolveu o gerador de AF, aparelho que forma o gás ozônio por meio de descargas elétricas em átomos de oxigênio. O tratamento com ozônio foi introduzido no Brasil no ano de 1975, pelo médico Heinz Konrad ${ }^{11}$.

O ozônio é um potente oxidante com efeitos antimicrobianos e fungicidas que, quando em contato com fluídos orgânicos, forma moléculas reativas de oxigênio, que por sua vez, proporcionam benefícios à reparação tecidual por meio de eventos bioquímicos do metabolismo celular ${ }^{11}$.

A ozonioterapia é uma técnica que utiliza o ozônio como agente terapêutico. No tratamento de feridas crônicas, sua utilização impulsiona a formação de tecido de granulação e neoangiogênese ${ }^{11}$.

$\mathrm{O}$ gerador de AF tem capacidade de produzir efeitos fisiológicos, como vasodilatação periférica, aumento do fluxo sanguíneo e consequentemente maior demanda de oxigenação e metabolismo celular, favorecendo o processo de cicatrização. Isso acontece devido aos efeitos térmicos que são produzidos por meio de corrente elétrica que atravessa o organismo, produzindo o efeito do ozônio pelo faiscamento que atravessa o eletrodo, eliminando os agentes patogênicos e oxigênio, responsáveis pela fibroplasia para elaboração de matriz intercelular ${ }^{10}$.

As indicações para o uso terapêutico do gerador de alta frequência e da ozonioterapia são diversas, sendo que existem indícios comprovando sua eficácia. Suas vantagens incluem potente ação antimicrobiana, fácil aplicação, baixo custo e ausência de efeitos adversos. Seus resultados clínicos tornam o aparelho de alta frequência um aliado para tratamento de lesões ${ }^{10}$.

Porém, apesar de todos os efeitos benéficos que qualificam o gerador de alta frequência como um recurso viável para tratamento de lesões, existe uma escassez de estudos científicos acerca de seu uso.

Diante do exposto, este estudo teve por objetivo relatar os efeitos no processo de cicatrização no tratamento da fasciíte necrotizante, com a utilização do gerador de $\mathrm{AF}$ em uma pessoa com diabetes.

\section{MÉTODOS}

Trata-se de um estudo descritivo, tipo relato de caso, desenvolvido em um ambulatório de feridas no interior de Minas Gerais, no período de agosto a outubro de 2018. Realizado com uma mulher de 52 anos de idade com diabetes mellitus tipo 2 e diagnóstico de fasciíte necrotizante.

Para avaliação da evolução da ferida, foi utilizada mensuração por meio de registro fotográfico com câmera de 12 megapixels com abertura $f / 2.2$, zoom digital de até 5 vezes e flash true tone. A análise das imagens foi realizada mediante o uso do programa Image J. A ferida foi avaliada no início do tratamento e reavaliada semanalmente a partir de então. A avaliação do processo de cicatrização da ferida foi efetuada por meio do instrumento Pressure Ulcer Scale Healing (PUSH).

Adaptado e validado no Brasil por Santos e Carvalho ${ }^{12}$, o PUSH é um instrumento utilizado para avaliar a cicatrização da lesão por pressão porque descreve as condições e a evolução das lesões, onde avalia a área da ferida, quantidade de exsudato presente na ferida e aparência do leito da ferida.

O item "área da ferida" é medido em centímetros quadrados e relaciona o maior comprimento (no sentido cefalocaudal) pela maior largura (em linha horizontal da direita para a esquerda). Os escores variam de 0 a 10 e os valores variam de 0 a $>24 \mathrm{~cm}^{2}$, conforme a área obtida ${ }^{12}$.

$\mathrm{O}$ item "quantidade de exsudato presente na ferida" é avaliado após a remoção da cobertura suja e antes da aplicação do curativo tópico. É classificado como ausente, pequena, moderada e grande e os escores variam de 0 (ausente) a 3 (grande) $)^{12}$. 
O item "aparência do leito da ferida" representa o tipo de tecido presente na ferida durante a avaliação e é dividida em: tecido necrótico (escara, cor preta), tecido desvitalizado (amarelo mucoide); tecido de granulação (coloração rósea ou vermelha); tecido epitelial (róseo ou brilhante) e tecido cicatrizado (ferida fechada). Os escores nesses tecidos são classificados como 0 (ferida fechada), 1 (tecido epitelial), 2 (tecido de granulação), 3 (esfacelo) e 4 (tecido necrótico) ${ }^{12}$

Ao serem somados os subescores dos itens, obtém-se um total com variação possível de 0 a 17 , sendo que os escores maiores indicam piores condições da ferida ${ }^{12}$.

Na primeira consulta, o histórico da paciente foi levantado a fim de iniciar o tratamento da ferida e ela foi convidada a participar da pesquisa. Durante as consultas seguintes, a paciente foi encaminhada para a sala de curativo. A limpeza da ferida foi realizada com irrigação de solução fisiológica 0,9\% morna e em jato e, após, a aplicação do gerador de AF foi realizada três vezes por semana com duração de 1 min nas margens e no leito, sobreposto a uma gaze estéril.

Como cobertura primária, gel de papaína a 2\% e ácidos graxos essenciais foram aplicados na região perilesional da ferida, e a lesão foi ocluída com gazes estéreis e fixação com micropore.

Os instrumentos de coleta de dados foram: ficha de caracterização para avaliação com dados sociodemográficos e clínicos, comorbidade, dados referentes à avaliação e à classificação das características da lesão. A avaliação da lesão foi realizada com base nos aspectos das feridas que compreendem três dimensões: tipo de tecido, quantidade de exsudado e área da lesão. Sequencialmente, o pesquisador realizou a perimetria da área da ferida com régua, captura das imagens e realizou o tratamento com AF.

Foi utilizado no estudo o gerador de AF marca Ibramed ${ }^{\circledR}$, portátil, bivolt 127 e 220 v, 50 e $60 \mathrm{~Hz}$, com dimensões de $7 \mathrm{~cm}$ de largura, $11 \mathrm{~cm}$ de profundidade e $6 \mathrm{~cm}$ de altura, e peso aproximado de $500 \mathrm{~g}$.

Foi utilizada técnica de faiscamento com intensidade média com eletrodo esférico maior. Ressalta-se que o material que entrou em contato com a ferida foi higienizado e desinfetado com álcool etílico hidratado 70\%.

O projeto de pesquisa foi submetido ao Comitê de Ética e aprovado sob o parecer 2.833.544, conforme preconiza a Resolução no 466 de 12 de dezembro de 2012, Conselho Nacional de Saúde (CNS), e a paciente convidada para o estudo assinou o termo de consentimento livre e esclarecido (TCLE).

\section{RESULTADOS}

C.H.S.R tem 52 anos, residente de Três Corações/MG, hipertensa, diabética, tabagista. Em julho de 2018, após uma sessão de depilação apresentou uma pústula na região infraglútea direita. Procurou atendimento ambulatorial com infectologista que indicou antibioticoterapia durante um mês. Após esse período, a paciente não apresentou melhora de seu quadro clínico, com agravamento gradativo da pústula, com rompimento e presença de hiperemia local, dor, pele descamativa e odor fétido. Ela foi encaminhada pelo infectologista para internação hospitalar, sendo diagnosticada pelo cirurgião local com fasciíte necrotizante e encaminhada ao desbridamento cirúrgico. Após o período de 11 dias de internação hospitalar, deu-se início ao tratamento ambulatorial da lesão.

$\mathrm{Na}$ avaliação inicial, a ferida apresentava uma área de 13,09 $\mathrm{cm}^{2}$, perilesional ressecado, margem descolada e irregular, cerca de $70 \%$ de tecido de granulação, $10 \%$ de esfacelo e 20\% de exposição de tecido subcutâneo (demostrados na Fig. 1), exsudato em quantidade moderada de aspecto seroso, cor amarelada e inodoro.

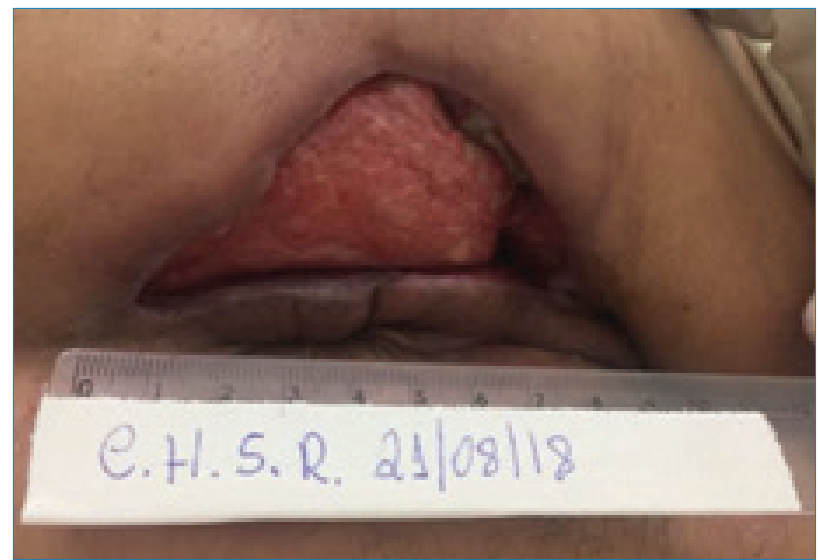

Figura 1. Lesão após a primeira aplicação.

Após sete aplicações, a lesão apresentava área de $4,58 \mathrm{~cm}^{2}$, perilesional íntegro, margem irregular, descolada, 95\% de tecido de granulação e 5\% de exposição de tecido subcutâneo, quantidade moderada de exsudato de aspecto seroso, coloração amarelada e inodoro, como apresenta a Fig. 2.

Após 15 aplicações, a lesão apresentava área de $1,94 \mathrm{~cm}^{2}, 95 \%$ de tecido de granulação, exsudato em pouca quantidade de aspecto seroso, coloração amarelada e inodoro. Em relação à área da lesão, a paciente apresentou melhora significativa no processo de cicatrização mensurado através do programa Image J de 13,09 para 1,94 $\mathrm{cm}^{2}$, como destaca a Fig. 3. 


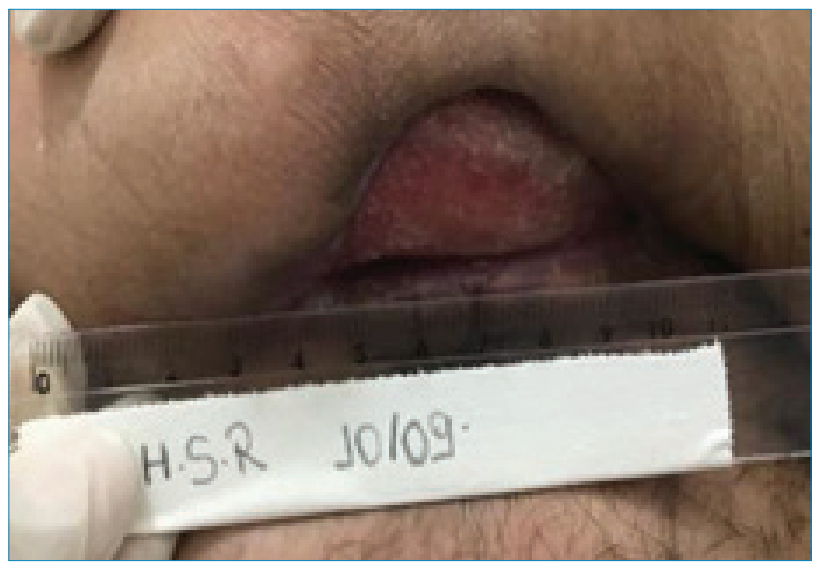

Figura 2. Lesão após sete aplicações.

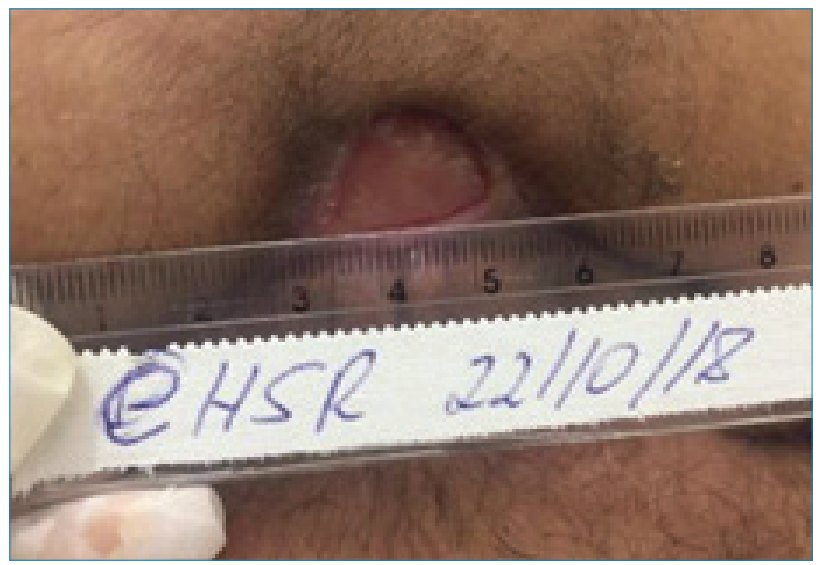

Figura 3. Lesão após 15 aplicações.

$\mathrm{Na}$ Fig. 4, estão representados os valores da escala de PUSH, utilizada para mensurar a área em centímetros quadrados, quantidade de exsudato e tipo de tecido presente na lesão.

Pode-se evidenciar que houve melhora significativa da lesão durante as seis semanas de avaliação. $\mathrm{Na}$ avaliação inicial, foi obtido PUSH escore 13, pois a ferida apresentava área de $13,09 \mathrm{~cm}^{2}$, quantidade moderada de exsudato e tecido de granulação em maior evidência. Nas três avaliações posteriores, foi verificada uma notória melhora da lesão, evidenciada na redução de sua área, que apresentou PUSH escore 7 na quarta avaliação, com área de $1,31 \mathrm{~cm}^{2}$, quantidade escassa de exsudato e tecido de granulação evidente. Na sexta e última avaliação, foi obtido PUSH escore 7, a área da lesão era de $1,94 \mathrm{~cm}^{2}$ com quantidade escassa de exsudato e tecido de granulação evidente.

No gráfico abaixo (Fig. 5), a área da lesão avaliada através do software Image Jé apresentada. Durante as quatro primeiras avaliações, foi obtida redução progressiva da lesão em $11,78 \mathrm{~cm}^{2}$, ou seja, $90 \%$ da área. Considerando a primeira e a última avaliação, foi obtida redução de $85,17 \%$ da área da lesão. Vale ressaltar que a falta de periodicidade da paciente nas duas semanas finais de tratamento pode ser indicativa para o aumento da área da lesão, pois houve aumento de $0,22 \mathrm{~cm}^{2} \mathrm{da}$ área.

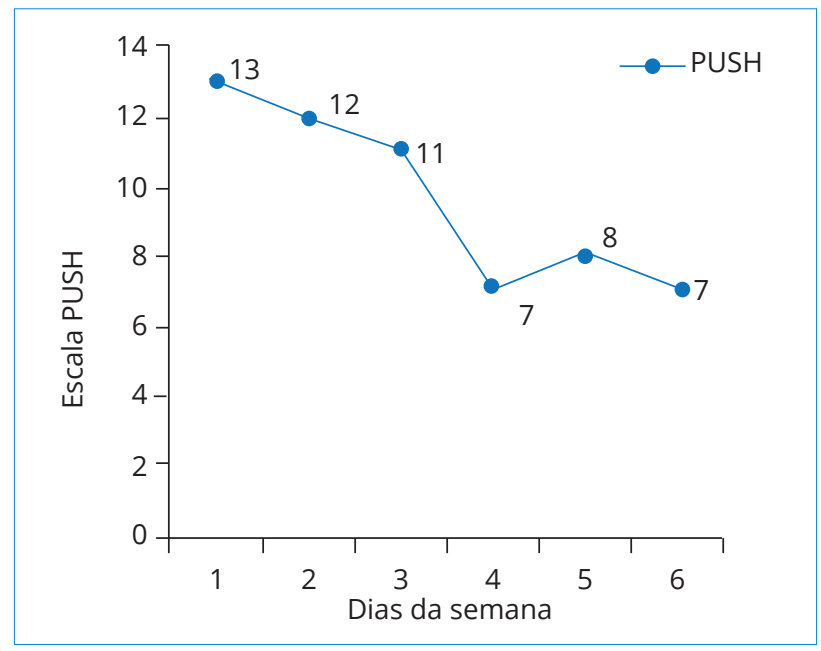

Figura 4. Escala de cicatrização PUSH.

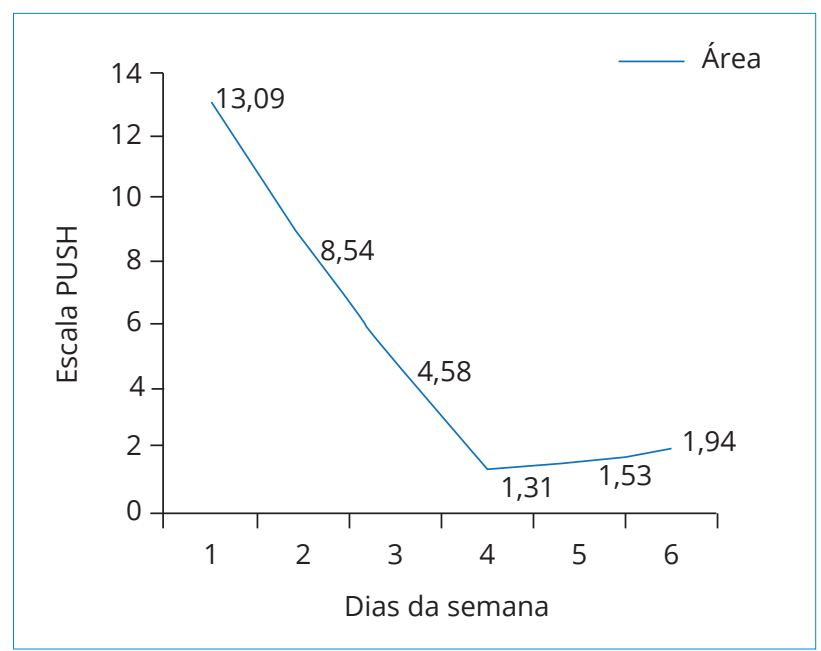

Figura 5. Área da lesão durante o tratamento.

\section{DISCUSSÃO}

Esse estudo teve como objetivo descrever o uso do gerador de AF no tratamento de uma ferida de fascíte necrotizante em uma paciente com diabetes mellitus tipo 2 , sendo que após as quatro primeiras avaliações foi obtida redução progressiva e significativa da lesão com quase $90 \%$ da área cicatrizada.

Outro estudo também relatou a efetividade do uso de gerador de AF com ozônio como recurso terapêutico no tratamento de feridas, em que obteve estimulação do processo de cicatrização e cicatrização total (62,2\%), seguidos 
da melhora do aspecto da ferida $(43,5 \%)$ e diminuição da dor $(17,4 \%)^{11}$.

A estimulação elétrica por AF promove a cicatrização de feridas, pois seu efeito térmico proporciona vasodilatação periférica, aumenta a oxigenação celular, promove a migração de células epiteliais, macrófagos e neutrófilos, além de estimular os fibroblastos a produzirem colágeno ${ }^{11}$.

Pesquisas experimentais com ratos apontam em seus resultados que a estimulação elétrica melhora a cicatrização de feridas por estimular fatores de crescimento na derme e na epiderme ${ }^{13}$. Estudo em humanos indicou que a estimulação elétrica pode ser uma intervenção efetiva no tratamento em diversas condições patológicas. Uma pesquisa descritiva realizada com três idosos com lesão por pressão demonstrou que a estimulação elétrica reduziu em 64,5\% a área da lesão de um indivíduo, sendo que nos outros dois ocorreu a cicatrização completa da lesão por pressão ${ }^{14}$. A estimulação elétrica em diabéticos, aplicada durante 12 semanas, levou à cicatrização completa das lesões ${ }^{15}$.

Outros dois estudos com o uso do gerador de AF para tratamento de lesão por pressão também se mostraram efetivos. No primeiro, após cinco semanas de tratamento com aplicação de AF três vezes ao dia durante $15 \mathrm{~min}$, utilizando técnica de faiscamento, houve redução no tamanho da lesão ${ }^{11}$. No segundo estudo, a técnica de faiscamento também foi utilizada, com amplitude de 80\% do aparelho durante 1 min por $\mathrm{cm}^{2}$ da lesão e houve melhora da cicatrização e diminuição de sua área ${ }^{10}$.

Há unanimidade entre diversos autores quanto ao uso de ozônio, seja aplicado por meio de óleo de girassol ozonizado, seja diluído em água, pois o ozônio propicia efeito antimicrobiano, bactericida e fungicida, além de sua eficácia na reparação tecidual. No tratamento de feridas crônicas, a utilização de ozônio induziu a formação de tecido de granulação, devido aos seus efeitos antissépticos ${ }^{11}$.

As feridas crônicas impactam em ônus para o Sistema Único de Saúde (SUS) e esse tema se tornou bastante discutido em fóruns econômicos de saúde devido ao uso exacerbado de recursos materiais e baixa resolução do problema. Portanto, o uso de recursos tecnológicos de forma sistematizada é de extrema importância, a fim de proporcionar a cura dos pacientes $^{10}$.

A enfermagem é uma ciência que promove prevenção e reabilitação por meio do cuidado. Portanto os profissionais devem utilizar tecnologias e recursos existentes, de forma a prestar uma boa qualidade de assistência. As tecnologias vêm sendo utilizadas na área da saúde em âmbito assistencial e educacional como forma complementar em metodologias de ensino ${ }^{10}$.

É essencial desenvolver estudos e processos tecnológicos de aplicação prática, para que o enfermeiro possa se manter atualizado. A tecnologia, quando utilizada no cotidiano da enfermagem, traz possibilidades para aprimorar o cuidado prestado, essencialmente à pessoa com ferida ${ }^{16}$.

\section{CONCLUSÃO}

A utilização do gerador de AF foi efetiva com base no resultado obtido, ou seja, com redução significativa da área da lesão, entretanto, é necessário que os achados evidenciados neste estudo sejam utilizados a fim de discutir e viabilizar medidas preventivas e de tratamento, e que sejam incentivadas novas pesquisas relacionadas ao tema, com maior número de pessoas, inclusive com outras abordagens metodológicas.

\section{CONTRIBUIÇÃO DOS AUTORES}

Conceitualização, Domingues EAR e Costa MHS; Metodologia: Domingues EAR e Costa MHS; Redação Primeira versão, Domingues, EAR, Costa MHS, Carvalho MRR e Kaizer UAO; Redação - Revisão \& Edição, Domingues EAR, Carvalho MRR e Kaizer UAO; Supervisão Domingues EAR.

\section{REFERÊNCIAS}

1. Klafke A, Duncan BB, Rosa RS, de Moura L Malta DC, Schmid MI. Mortalidade por complicações agudas do diabetes melito no Brasil, 2006-2010. Epidemiol Serv Saúde. 2014;23(3):45562. https://doi.org/10.5123/S1679-49742014000300008

2. Soares FG, Pessanha ALP, Lemos LVB. Fasciíte Necrotizante em paciente diabético. Rev Cient Fac Med Campos. 2013;8(1):28-31.
3. Conselho Federal de Enfermagem. Resolução COFEN No 570 de 09 de março de 2018 [Internet]. [Citado 13 set 2019]. Disponível em: http://www.cofen.gov.br/resolucao-cofenno-0570-2018_61172.htm

4. Yamada BFA, Ferrola EC, Azevedo GR, Blanes L, Rogenski NMB, Santos VLCG. Competências do Enfermeiro Estomaterapeuta 
(ET) ou do Enfermeiro Pós-graduado em Estomaterapia (PGET). Estima, Braz J Enterostomal Ther. 2008;6(1).

5. Brasil. Lei $n^{0} 7.498$ de 25 de junho de 1986. Que dispõe sobre o Exercício profissional da Enfermagem, e dá outras providências. [Citado 13 set 2019]. Disponível em: http://www.cofen.gov.br/lei-n-749886-de-25-de-junhode-1986_4161.htm

6. BRASIL. Decreto $n^{\circ} 94.406$ de 08 de junho de 1987. Que regulamenta a Lei $n^{\circ} 7.498$ de 25 de junho de 1986, que dispõe sobre o Exercício profissional da Enfermagem, e dá outras providências. COFEN. [Citado 13 set 2019]. Disponível em: http://www.cofen.gov.br/ decreto-n-9440687_4173.html

7. Conselho Federal de Enfermagem. Resolução COFEN No 358 de 15 de janeiro de 2009 [Internet]. [Citado 13 set 2019]. Disponível em: http://www.cofen.gov.br/resoluocofen-3582009_4384.htm

8. Conselho Federal de Enfermagem (Brasil). Resolução COFEN n 567 de 29 de janeiro de 2018. [Internet]. [Citado 03 mar 2018]. Disponível em: http://www.cofen.gov.br/ resolucao-cofen-no-567-2018_60340.html

9. Morais GFC, Oliveira SHS, Soares MJGO. Avaliação de feridas pelos enfermeiros de instituições hospitalares da rede pública. Texto Contexto - Enferm. 2008;17(1):98-105. https://doi.org/10.1590/S0104-07072008000100011

10. Korelo RIG, Oliveira JנJ, Souza RCA, Hullek RF, Fernandes LC. Gerador de alta frequência como recurso para tratamento de úlceras por pressão: estudo piloto. Fisioter
Mov. 2013;26(4):715-24. https://doi.org/10.1590/S010351502013000400002

11. Oliveira LMN. Utilização do ozônio através do aparelho de alta frequência no tratamento da úlcera por pressão. Rev Bras Ciên Saúde. 2011;9(30):41-6. https://doi.org/10.13037/ rbcs.vol9n30.1418

12. Santos VLCG, Carvalho VF. Reapresentando o instrumento pressure ulcer scale for healing (PUSH) para avaliação de úlceras por pressão e úlceras crônicas de perna. Estima, Braz J Enterostomal Ther. 2009;2(2).

13. Kutlu AK, Çeçen D, Gürgen SG, Sayin O, Çetin FA. Comparison study of growth factor expression following treatment with transcutaneous electrical nerve stimulation, saline solution, povidone-iodine, and lavender oil in wounds healing. Evid Based Complement Alternat Med. 2013;(1):1-9. https://doi. org/10.1155/2013/361832

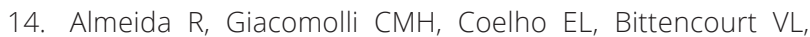
Callegaro CC, Stumm EMF. Gerador de alta frequência no tratamento de lesão por pressão em idosos. Rev Enferm UFPE On Line. 2017;11(8):3136-142.

15. Barnes R, Shahin Y, Gohil R, Chetter I. Electrical stimulation vs. standard care for chronic ulcer healing: a systematic review and meta-analysis of randomised controlled trials. Eur J Clin Invest. 2014;44(4):429-40. https://doi. org/10.1111/eci.12244

16. Queiroz PES, Schulz RS, Barbosa JDV. Importância da tecnologia no processo de enfermagem para o tratamento de feridas crônicas. Rev Enferm Contemp. 2017;6(2):158-166. 